\title{
Intelligent System to Find the Health Care Centers for Senior Citizens Based on Disease and Nearest Locations using GPS
}

\author{
Gurram Sunitha $^{a}$, Sasikumar Gurumoorthy ${ }^{\mathrm{b}}$, E.S. Madhan ${ }^{\mathrm{c}}$, R. Reeba ${ }^{\mathrm{d}}$, and L P Supriya ${ }^{\mathrm{e}}$ \\ Professor, SreeVidyanikethan Engineering College, Tirupati, India \\ brofessor, SreeVidyanikethan Engineering College, Department of CSE, Tirupati, India \\ 'School of Computing, SRM Institute of Science and Technology, SRM Nagar, \\ Kattankulathur,Chengalpattu District, Tamil Nadu-603203, INDIA. \\ dAssistant Professor, Sreebhudha college of Engineering, pattoor, allapuzha, kerala \\ eAssistant Professor, Department of computer science, Sreebhudha college of Engineering pattoor, allapuzha, kerala.
}

Article History: Received: 11 January 2021; Accepted: 27 February 2021; Published online: 5 April 2021

\begin{abstract}
It is not convenient nowadays for senior citizens to find an accessible and appropriate health care centre that can meet their needs. Therefore, this initiative helps senior citizens to look for a suitable facility depending on their location and their current illness. The scheme is simple and easily produces results. By using GPS feature or Internet network by using position coordinates and pre-built coordinate databases of nursing care facilities, the details of the nearest facility can be obtained along with the name of the location and buildings with smart phones. The software also provides a tracking service for consumers (senior citizens) so that, by forming a technical relationship with medical centres, it can offer a range of highquality facilities. The contribution of this system is that through the $\mathrm{O} 2 \mathrm{O}$ service, emergency responders can intelligently find a correct hospital that can treat the illness or accident of patients with a smart phone.
\end{abstract}

Keywords: Elderly nursing care Facility search, User's location, Disease based search, Intelligent System, Health Care Centers.

\section{Introduction}

The number of healthcare care facilities is rapidly increasingly growing. The suggested framework searches for a suitable facility for the patient based on the position of the user and his / her current illness[1]. The scheme is simple and easily produces results. With smart phones, using the embedded GPS feature or the Internet network, you will find your current location. The data of the closest facility can be accessed by using the position coordinates and pre - built coordination database of nursing care facilities. When elderly people are put in an emergency situation, paramedics can briefly become confused as they are unable to understand the medical details or background of their patient as doctors can, especially when they do not know their identity[2]. For specific diseases such as cardiac diseases, the patient's fate can depend on their swift decisions. The location of community clinics, health care centres and city hospitals are decided by certain applications. The importance of this method to the system [3].

Location-based services give smart phone users many advantages in gathering information regarding the current location and analysing the information to become more valuable information close to their location. Location-based solutions can be deployed on Android-based mobile phones with the aid of A-GPS on phones [4] and via Web Services using GPRS to provide such value-added services: informing clients of local traffic conditions, offering routing information, helping clients locate nearby hotels[5]. In this article, through Google Web Services and Walk Score Travel APIs on Android phones, we suggest the introduction of location-based services to offer users multiple services based on their location.

Smartphone produces an Android operating system (AOS) widely used since it is an open access software Linux distribution and developed specifically for mobile touchpad devices like smart phones and tablets. Many implementations use internal Mobile hardware, particularly sensors such as proximity, accelerometer and gyroscope detectors, to react to explicit user actions [6]. Different applications (apps) are downloadable from the Google Play web retailer.

Millions of extra applications are uploaded to its online database every day. In the medical group, a variety of healthcare-related applications are available which are useful in the diagnosis of heart rhythm parameters, vaccine schedule, medication recall, etc. Mobile-health (m-health) distinguishing proof in these kinds of apps. The location of health clinics, healthcare centres and city hospitals is decided by certain requests. The unique concept of this application is to look for the closest specialty hospital in the city of Karachi [7]. There is authentic and upto - date data about of hospital admissions available. Below is the context details regarding selecting this application's AOS system.

1.1 Problem Statement

The suggested system is a type of system that provides data on health care facilities depending on the level and illness of the patient. The paramedics can briefly become confused when an elderly person is put in an 
emergency matter, as they do not recognize the medical details or background of their patient as doctor can, particularly when they do not know their identification. For specific diseases such as cardiovascular disorders, the patient's fate can depend on their swift decisions. The benefit of this method is that with a mobile phone, emergency responders can intelligently identify a correct hospital that can handle the illness or accident of victims.

\subsection{Motivation}

Recent times, due to a sharp increase in the number of senior citizens, the older population that needs healthcare services or nursing facilities has been growing rapidly in the Republic of Korea. But having an open and appropriate health care centre that can meet their needs is not simple for them. An elderly with a cardiovascular disease, for example, must be near to a medical centre that can take care of his / her illness. As the independence of seniors is restricted, this also refers to other diseases. The software developed in this work therefore enables senior citizens to find a suitable treatment clinic by using GPS-based location data from smartphones along with a web push system [8].

1.3 Objectives

o Senior Citizens can search the hospitals nearby based on their disease

o He can book the appointment to that hospital if it is a normal check up based on the available slots in the hospital

o With the help Global positioning System (GPS) user can easily find the route to the hospital easily

o In the case of emergency patient can be admitted to nearby hospitals based on the distance we get.

1.4 Significant Contributions from Investigation

GPS facility for location access [9]

Online firebase database

Google map application program interface

- $\quad$ Easy appointment booking for senior citizens

\section{Literature Survey}

The advances in medicine and engineering, medicine and global safety, combined with improved knowledge of nutrition and ecological and public hygiene, have prepared the path for the significant increase in life expectancy globally over the past several decades, the authors of[1] suggested. Increased health expectancy, however, has given rise to a rising ageing population, thus jeopardising many countries' socio-economic system in terms of costs connected with elderly health and well-being. It is important to establish affordable, unfussy and easy-to-use health care in trying to adapt with the rising demand for elderly health services.Home automation, which incorporate environmental and general adaptation sensors, actuators, and advanced information and communication technology, will make it possible to monitor elderly safety and well-being continuously and remotely at a low cost. Instead of costly and small healthcare facilities, smart homes will allow the elderly to live in their comfortable home environments. Healthcare staff also can keep track in near real - time of the elderly's overall health status and provide feedback and support from remote facilities. In the this paper, we presented a thorough analysis of state-of-the-art research and innovation in remote healthcare technology focused on smart homes.

Authors of [2] proposed the study intends to quantify the changes and transformations in features classes of Yamuna River Flood Plain in Delhi. ERDAS imagine 9.2 and Terrset geospatial software were used for image processing and quantitative assessment, transformation, gain and loss, contribution o net change and spatial trend analysis. The Landsat 8 (2018), TM (2000) and MSS (1989) images were acquired for assessing LULC change detection using Maximum Likelihood Classifier. LULC classification was achieved with kappa coefficient and overall accuracy for Satellite images of MSS (1989), TM (2000) and Landsat 8 (2018) as 0.781, 0.892 and 0.804 and $86.00 \%, 92.31 \%, 86.00 \%$ respectively. Analysis reveals the addition of built up area up to $25 \%$ from year 2000 onwards and loss in dense forest from $40 \%$ to $30 \%$. Vegetation areas recorded a reduction of $15 \%$ from 1989 to 2000 . Spatial trend reveals the qualitative vulnerability of vegetation classes during the study period. During 1989-2000, dense forest, vegetation and water classes contributed maximum to settlement class and during 2000-2018 an interchange of dense forest and vegetation was witnessed. The study provides an insight to the sustainable planning and management of the river ecosystem that is affected by population expansion.

The writers of [3] proposed a system for senior citizens in need of healthcare services or nursing services, in particular for the elderly. But having an open and appropriate healthcare centre that can meet their needs is not easy for them. An elderly with a cardio vascular disorder, for example, should be near to a medical centre that can care for his / her condition. As the independence of seniors is limited, it also applies to many other diseases. Thus, in this report, the programme's systems helps old people to find a suitable medical facility by using GPSbased location data from smartphones along with a mobile push device. Also, with both the Bluetooth beacons, this $\mathrm{O} 2 \mathrm{O}$ software tracks the user. The development plan independently calculates the user's current location with a GPS feature or authentication tokens system to identify from the database the closest medical centres or clinics where information about the position of medical facilities and users, along with their professions, has been processed in preparation. To define relevant prospective healthcare centers, the straight-line formula and the user 
coordinates are used. The software also provides a tracking service for consumers (senior citizens) so that, by forming a technical relationship with treatment clinics, it can offer a range of high-quality facilities.

Writers of [4] suggested digital equipment based on android platform (AOS) such as mobile computers or mobile tablets are commonly used in many uses, such as text messenger, gaming, internet browsing, networks and various application code download. In android Smart phones, there are numerous types of healthcare apps that help family caregivers minimise time and cost effectiveness. This project suggested a new framework concerning the hospital management system that addresses our day-to-day needs. Based on their criteria, the patient can scan the clinic. This application includes information of the hospital and physicians available based on the patient application. The generalisability is created to locate the closest town with the required specialist doctor in a radius of about five $\mathrm{km}$. With a built-in Global Positioning System function in cell devices, the nearest position of clinics is determined and finds the path via Google Map Program Interfaces (API) from the present location. Based on specialised consultant availability, a patient can grab the closest hospital with the aid of this application. A detailed profile of doctors and hospitals, such as the website, mailing addresses and phone numbers, are available in the application. The established android app is a user-friendly provider that enables patients with the necessary information in an effective way.

The writers of [5] suggested a software development approach to help people with chronic. Diabetes is closely linked to the eating habits and lifestyle that often cause hyperglycemia so that it is important for diabetics to adjust them in order to improve or manage the main complications of dementia The solution-designed software allows patients to easily identify their sugar levels and available treatments regimens and then suggests a more effective approach together with the solution The main purpose of this research is to help diabetes clinicians by supplying patients themselves with a comfortable but efficient means of avoiding or enhancing diabetic symptoms, which will improve the lifestyles in such a positive way in effect.

The writers of [6] suggested a system of collective recommendation. Things based on differences between user tastes are recommended by collective recommendation systems. This paper presents a system of collective guidelines that proposes elective university courses for students by taking advantage of courses taken by other similar students. As an overarching strategy to discover trends between courses, the proposed method employs an association rules neural network. In order to determine the overall performance of the proposed solution, assays were conducted with real - world data sets.

The writers of [7] suggested a method of monitoring system of mass fish mortality. For the operation and maintenance of preparation, a consistent supply of electric power at Fish Farms is important.The device suggested is autonomous and easy enough for the workers to work. Some questions about the PLC technologies being applied as it also causes the loss of cognitive behavioural therapy.

Middleware extensions that provide a versatile, scalable approach to distributed application development were proposed by the authors of [8].Middleware interfaces for distributed computing have been developed by researchers from Cambridge University. A versatile, flexible approach to the creation of application components is provided by middleware interfaces.

\subsection{Limitations of Existing Method}

- All senior citizens may not be aware of usage of smart phones

- IOS users cannot use this application it is limited to android. So IOS users

- Public health care companies have long worried about the privacy and protection of data. This was the reality before data, thanks to mobile phones and other devices, like laptops , tablets, and hard disk drives, could move so freely beyond their facility

- $\quad$ is as it can now. The files contained on it and available through apps can be accessed and probably downloaded if a smart phone is stolen or lost and lacks sufficient levels of protection. For an agency and clinician, such a situation may have important financial and legal consequences.

- Although not all mobile device apps need access to the Internet to work efficiently or to their maximum potential, many do. Thankfully, there are also plenty of cell towers and many sites that provide Wi-Fi connectivity. Neither, however, is guaranteed, because if a smart phone has been unable to receive and transmit data, some apps cannot work as expected.

- Price, with new smart phones usually running at least many hundred dollars, and also some models reaching $\$ 1,000$, is a major barrier to smart phone acceptance. Such high prices often contribute to holders hanging onto their appliances for long..

\section{Procedure Adopted}

A concept of a mobile medical facility search functionality for elderly people based on venue. This system, whereas, is effectively implemented by the Bluetooth feature and the gyroscope (data link layers and physical layers) and GPS installed on the smart phone (transport layer), that will then enforce the actual service for senior citizens residing alone via its intelligent Java-based mobile medical facility search application (application layer) [15]. A mobile medical facility regional system depending on the level and illness of the patient is proposed in this section. After requesting a patent, this device will be optimised for both Android operating systems as a personal app service. The spectrum of mobile healthcare facilities search system development is shown in Fig. 1. 


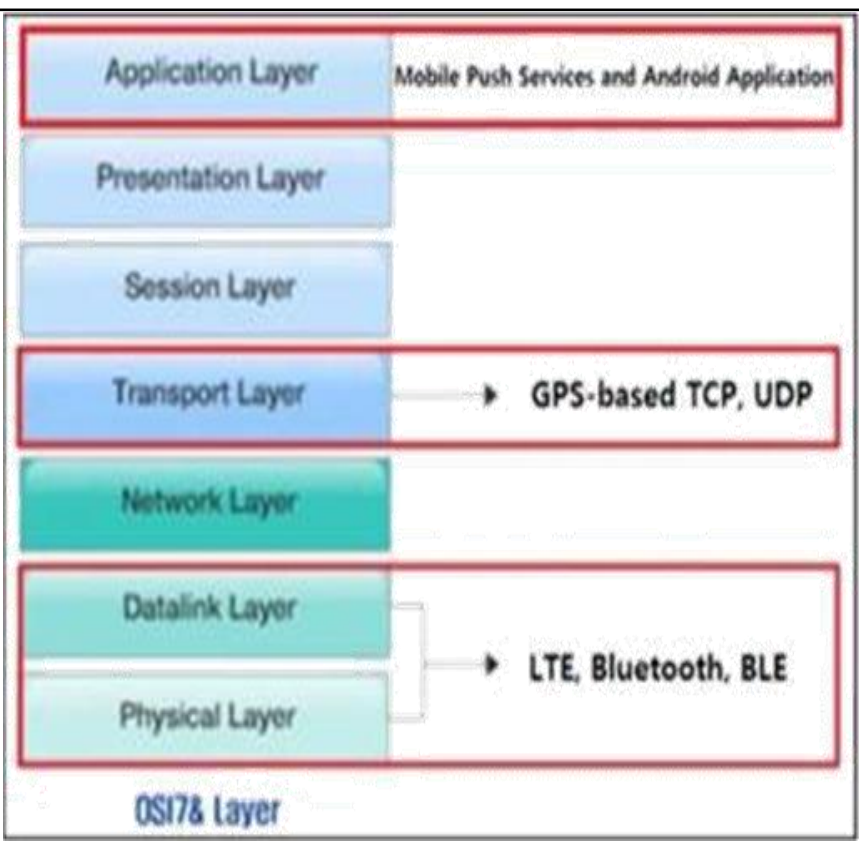

Fig. 1 Mobile health care facility search system's development scope

An implementation of location-based mobile health care facility search system for senior citizens applicationSuch that, it will become the ultimate user interface (UI) in which it collects and shows location information. By using GPS and the system alone, the phone can find current locations so that it obtains the coordinates of centres and houses in advance and stores them in the database to estimate the position of a nearby centre based on the location and the single edge equation [16]. It is necessary to record all the data in the database and use the Firebase application. These are correlated with the broad Key Operation database keys.

\section{Implementation}

4.1 Implementation of Location Based Services

LBS Application Programming Interfaces (APIs) support Android. The position service enables the present position of the unit to be identified. The program can request that system location data be updated periodically [17]. For distance warnings such as when the system enters and exists from a region of defined longitude, latitude and distance, the software may also register a purpose receiver.

Android Location API

These are the dissimilar classes existing under Location API bundle to recover the Location data of the worker.

Location Manager- The class deliversentree to the location provision. It also deliversamenities to get the finest Location Benefactor as per the standards.

Location Provider-It's an intellectual super class for position providers. A location breadwinner providesintermittentintelligences on the geographical position of the device.

Location Listener- This class deliverscall backapproaches which are named when location getsdifferent. The hearer object has to be listed with the position manager.

Criteria- The class delivers the request to choose appropriate Location Provider by as long asadmission to a set of compulsorybelongings of the Location Breadwinner.

The Google Places API is a facility that earnings data around Places - distinct within this

Web Service as, three-dimensional locations, or favoured points of interest — using HTTP requests.

Place answer specifies sites as latitude/longitude organizes.

The four kinds of needs are obtainable finished the Google Places API-

- Place Searches - It returns acollection of neighbouring Places founded on a location distinct by the userPlace hunt API dispute is exposed in Table 3.1

- A Place Huntappeal is an HTTP URL definite in the subsequent way:

- https://maps.googleapis.com/maps/api/place/search/output?arguments

- Where production may be moreover of the following standards

- Json demonstrations the comeback in JavaScript Object Notation (JSON)

- $\quad \mathrm{xml}$ demonstrationsproductivity as XML.

Table 1 Place search API argument 


\begin{tabular}{l|l} 
Arguments & Description \\
\hline $\begin{array}{l}\text { Location } \\
\text { (required) }\end{array}$ & $\begin{array}{l}\text { The latitude/longitude about which place } \\
\text { information is to be found out. This must } \\
\text { be defined as latitude, longitude. }\end{array}$ \\
\hline $\begin{array}{l}\text { Radius } \\
\text { (required) }\end{array}$ & $\begin{array}{l}\text { Distance (in meters) about which to show } \\
\text { Place results. }\end{array}$ \\
\hline types (optional) & $\begin{array}{l}\text { Limit the results to places matching at least } \\
\text { one of the pre defined types. Types must be } \\
\text { separated with a pipe notation } \\
\text { (type1ltype2letc). }\end{array}$ \\
\hline $\begin{array}{l}\text { Language } \\
\text { (optional) }\end{array}$ & $\begin{array}{l}\text { The language code, showing in which } \\
\text { language the results must be shown, if } \\
\text { possible. }\end{array}$ \\
\hline name (optional) & $\begin{array}{l}\text { A term to be mapped against the names of } \\
\text { Places. Results will be limited to those } \\
\text { having the name. }\end{array}$ \\
\hline $\begin{array}{l}\text { sensor } \\
\text { (required) }\end{array}$ & $\begin{array}{l}\text { Indicates whether or not the place request is } \\
\text { from the device having a location sensor }\end{array}$
\end{tabular}

Place Details - It revenues more exact data around a user distinct Place.

Place Check-ins - It permits the appeal that anindividual has check in to a Place. Check-ins iscast-off to instrument a Place's admiration.

Place Reports - It permits the operators to add new positions to the Residence service, and to delete Spaces that the claim has supplementary to the catalogue.

JavaScript Object Notation of location Information in database:

Location information of hospitals are stored in the database in the procedure of latitude and longitude.

Format of JSON object is:

\{

"address" : " National Highway, 71, Renigunta Road, SV Auto Nagar, Tirupati, Andhra Pradesh 517506 ",

"hospitalName" : "Narayanadri hospital",

"location" : "13.629409,79.463720",

"speciality" : "Multispeciality",

"timings" : "24/7"

\} ,

\{

"address" : " T. Nagar, near Sri raghavendraSwamyMatam, Tirupati, Andhra Pradesh 517501 ",

"hospitalName" : "Sri Padmavathi Multi Specialties hospital",

"location" : "13.640474,79.426520",

"speciality" : ["NEURO","Multispeciality"],

"timings" : "24/7"

\} ,

\{

"address" :"Sri PadmavatiMahilaVisvavidyalayam, Tirupati, Andhra Pradesh 517507",

"hospitalName" : "SVIMS CANCER center",

"location" : "13.637127, 79.404193",

"speciality" : "CANCER CENTER",

"timings" : "24/7"

\} ,

\{

"address" : " Cherlopalli - Alipiri Rd, Sri PadmavatiMahilaVisvavidyalayam, Tirupati,Andhra Pradesh 517502 ", 


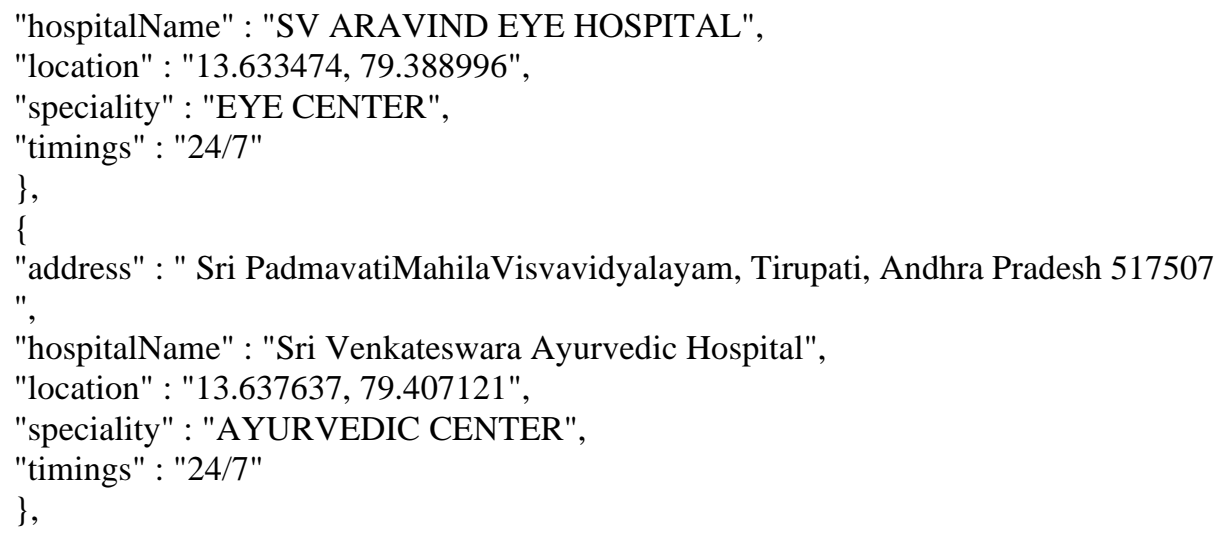

4.2 Book The Appointment In Hospitals

Using the GPS, and data present in the database all the hospitals are listed based on the distance, specialization.

Now user (Senior citizen) can be able to book the appointment in the hospital based on the slots available. In case of emergency senior citizen can directly admit in the hospital. This feature makes senior citizens easily makes the appointment so that they cannot wait for a longer time to get the appointment [18]. They can reach the hospital at the time of appointment. When the appointment is booked senior citizen can see the status of whether the appointment is accepted or rejected by the hospital. All the appointments of the user will be displayed in hospital login. Hospital admin can cancel or accept the appointment.

\section{Physical Model}

The basic flow of the application and physical model of an android application and life cycle of an application is shown in Fig.2. Activity consists of all the screens of our application. 


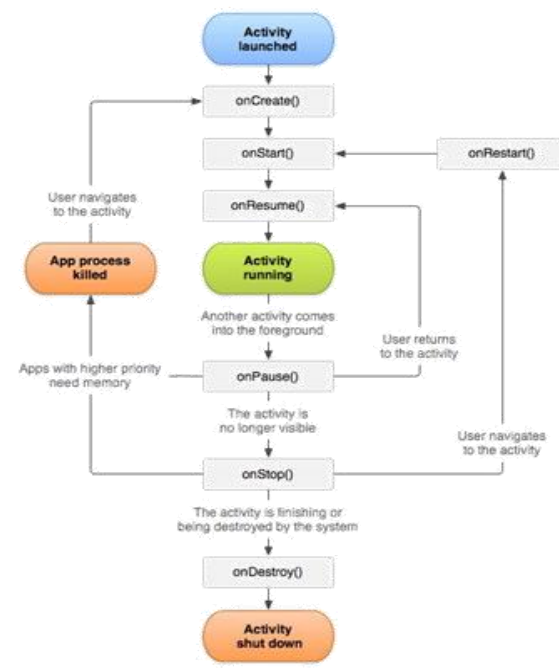

Fig. 2 Physical Model

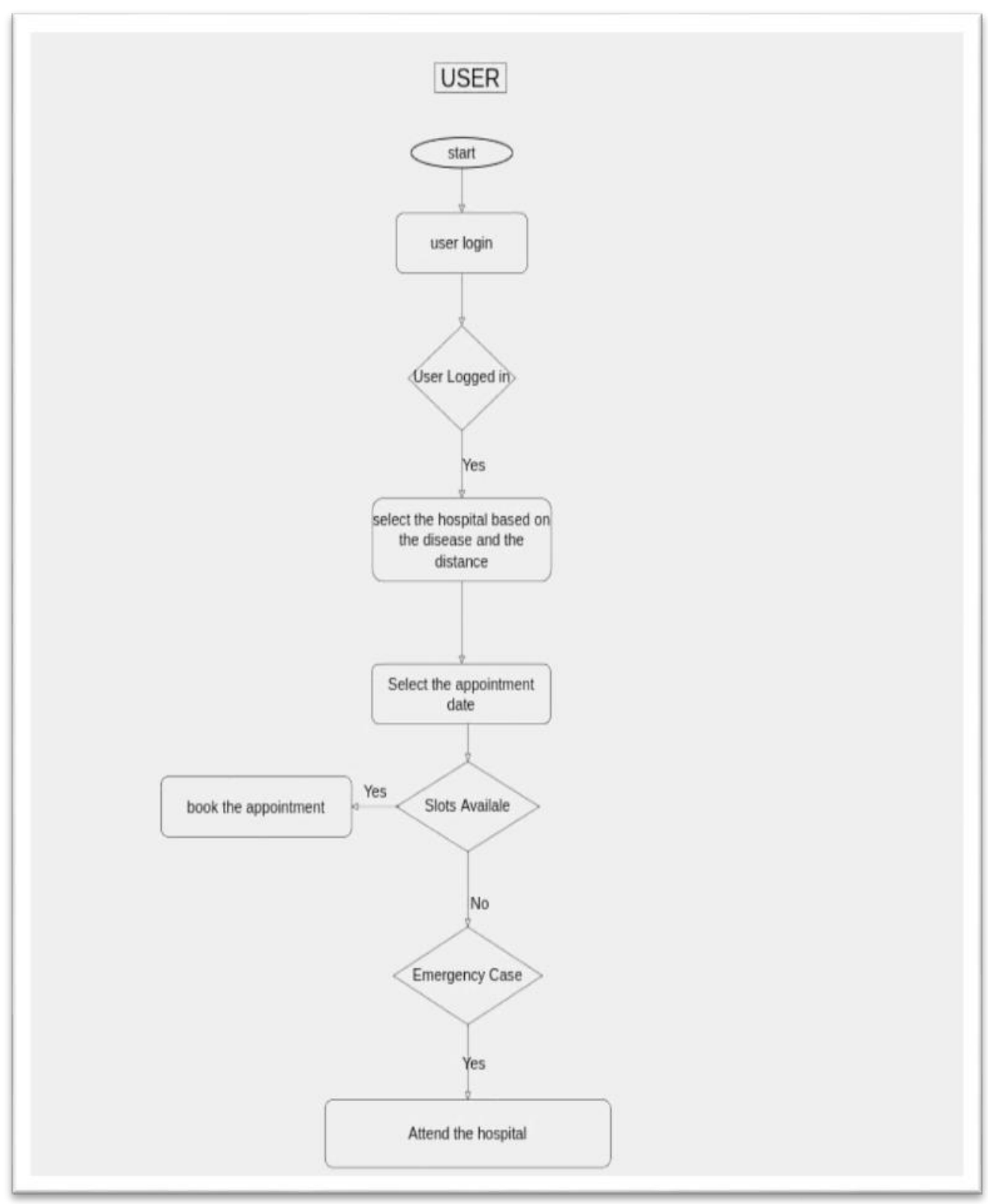

Fig.3 Simulation Flow

5.1 Simulation Model

Simulation model represents the basic flow of the application i.e., how the application flow. Simulation model is shown in Fig.3. 


\section{Results}

The results of the application are:

1. When the application icon is pressed on the smart phone, the application will be opened and it will show a splash screen and then the home screen containing all the hospitals listed distance wise.

2. When the specialty or the category is pressed, it will display the hospitals based on the specialization.

3. When the specific hospital is selected, this will show the hospital specific page with the details of the hospital and appointment booking page.

4. When the date, slot, and the booking appointment is selected the appointment will be booked and it will be sent to the hospital.

5. When the request is sent to the hospital, the hospital can accept or cancel the appointment.

6. If the appointment is accepted the patient will get the appointment as active and he/she can approach the hospital.

7. If the appointment is cancelled then the patient can approach another hospital.

8. If the patient needs direction to the hospital show directions button will redirect to google maps Results of output screens are shown below

Fig. 4 shows the login screen when the application is opened
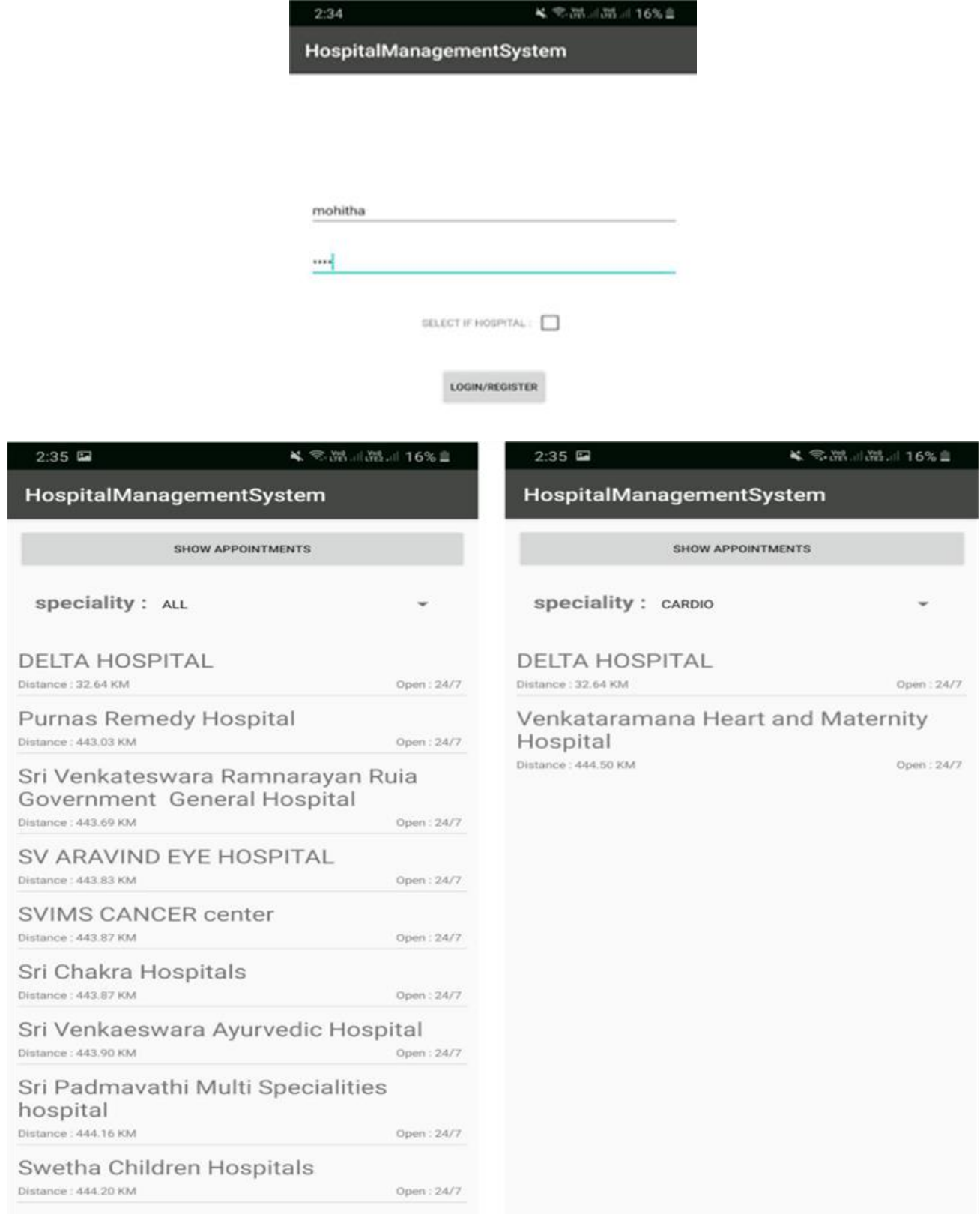

Fig.5 Home splash screen \& Hospital list based on disease

- Hospital home screen and hospitals lists based on the disease is shown in Fig.5.

- When you select the hospital appointment booking screen is displayed which is shown in Fig.6 


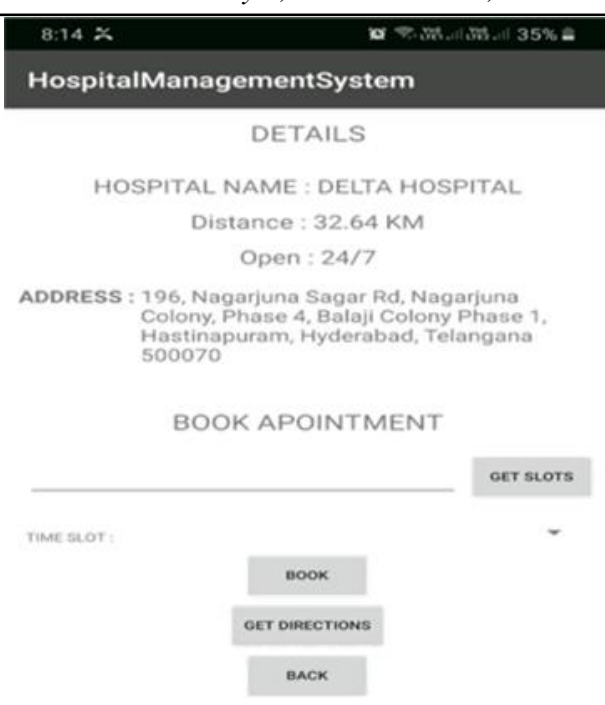

Fig.6 Appointment booking of selected hospital

If the user does notknow, the directions to the hospital user can click on get directions it will directly redirect to Google map screen, which shown in Fig.7.

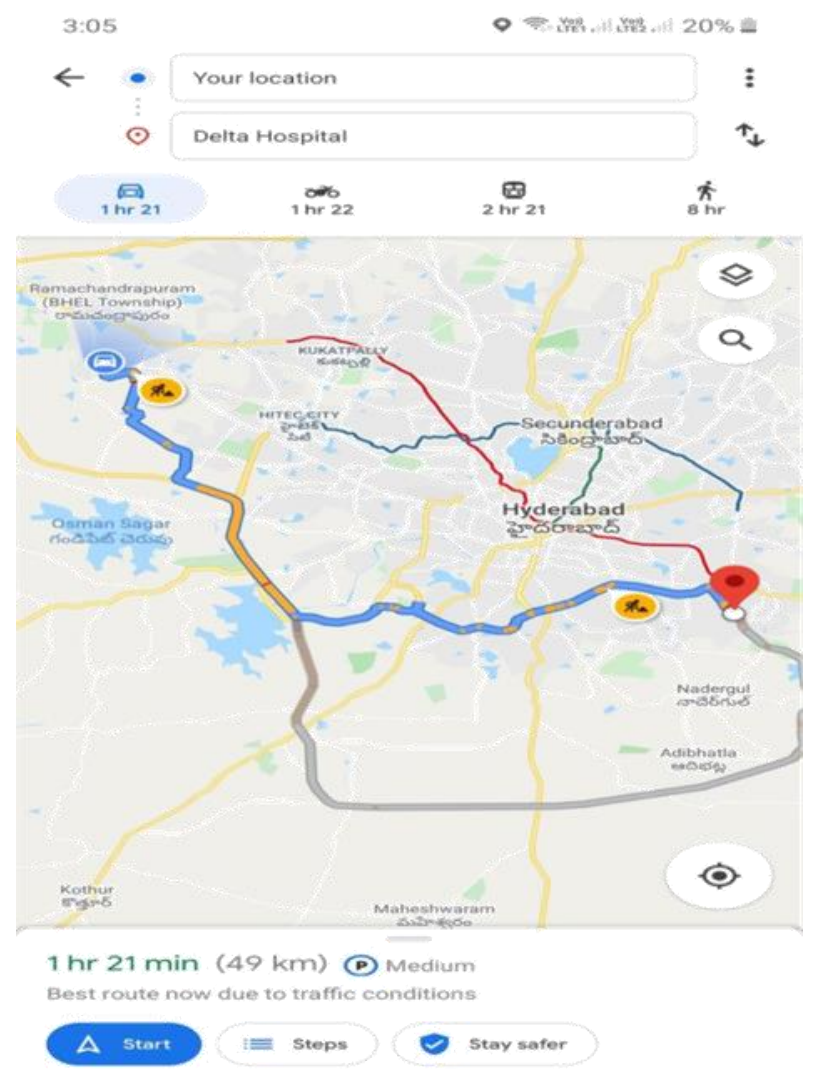

\section{Conclusion}

Fig.7 Map display screen to show directions

The suggested framework offers information on nursing care facilities depending on the level and illness of the patient. The fire fighters can briefly become puzzled when an older person is put in an urgent situation, as they cannot know the medical information or background of their client as doctor can, particularly once they do not recognize their name. For various cancers such as cardiovascular disorders, the patient's fate can rely on their swift decisions. The benefit of this device is that through GPS, medical responders can smartly locate a correct facility that can treat the illness or accident of patients with a smart phone. In emergency situations and also for the non-resident individual of the region, this software is highly useful. In a single application, the specialised list of physicians in each clinic is often useful for future consultations. 


\section{Future Scope}

The future scope of this application is to establish and assess, on a real-time basis, the availability of a specialist doctor near the location of the patient. This advancement would also provide an opportunity to book online appointments to make it easier for patients to save time.To be more adequate for our goal, we intend to refine the framework. We intend to connect the scheme with other applications concerned with other fields. In our future scheme, a new marketing approach and service spirit will be emphasised.

\section{References}

1. Kim, T-J et al (2017) An elderly nursing care facility search system based on user's location and disease. In: Proceeding of MUE 2017, p 1

2. Wu S-J, Chiang R-D, Wu T-F (2018) Direct mail promotion mechanisms and their application in supermarkets. J Supercomput 24:1-18

3. Lee S-G, Cha E-Y, Sung Y (2015) Impulse noise filter for beacon-AP signal processing in real time.Converg 6(4):1-7

4. Moon SY, Park J-H A design of scheduling program for diabetic patients, a software engineering approach

5. Sung Yu, Kwak J, Park JH (2015) Graph-based motor primitive generation framework. Hum Cent ComputInfSci 5(35):1-9

6. Park J et al (2015) Design of the real-time mobile push system for implementation of the shipboard smart working. In: Advances in computer science and ubiquitous computing, LNEE (CUTE 2015), Springer, vol 373, pp 541-548

7. Huh J-H, Kim N, Seo K (2016) Design and implementation of mobile medication-hour notification system with push service function. Int J ApplEng Res 11(2):1225-1231

8. Bacon J et al (2000) Generic support for distributed applications. IEEE Comp 33(3):68-76

9. Bhide M, Deolasee P, Katkar A (2002) Adaptive push-pull: disseminating dynamic web data. IEEE Trans Comput 51:652-668

10. Bessis N, Asimakopoulou E, Conrad M (2009) A mathematical analysis of a data-grid push service for disaster management response scenarios. In: Proceedings of International Conference of Computing, Engineering and Information, pp 8-11

11. Podnar I, Hauswirth M, Jazayeri M (2002) Mobile push: delivering content to mobile users. In:Proceedings of the 22nd International Conference on Distributed Computing Systems Workshops (ICDCSW'02), pp 563568

12. Sutton P, Arkins R, Segall B (2001) Supporting disconnectedness-transparent information delivery for mobile and invisible computing. In: First IEEE/ACM International Symposium on Cluster Computing and the Grid, IEEE, pp 277-285

13. Huh J-H, Seo K (2016) Design and test bed experiments of server operation system using virtualization technology. Hum Cent ComputInfSci 6(1):1-21

14. Álvarez-Bermejo J-A, Belmonte-Ureña L-J, Estrella-Ramón A (2016) An innovative technology proposal for the improving in the communication, social reputation and service quality: a case applied to the hospitality sector. El profesional de la información, vol 25, No. 6, pp 960-969

15. Álvarez JA, Lodroman MA, López-Ramos JA (2015) A blind signature-based protocol for tracing traitors. Int J Comput Math 92(9):1764-1774

16. Choi J-H, Huh J-H, Weon S (2017) A design of scheduling program for diabetic patients: a software engineering approach. In: LNEE, MUE 2017, Springer, pp 1-6

17. Huang Y, Garcia-Molina H (2001) Publish/subscribe in a mobile environment. In: Proceedings of 2nd ACM International Workshop on Data Engineering for Wireless and Mobile Access (MobiDE'01),(Santa Barbara, Calif.), pp 27-34

18. Bacon J et al (2000) Generic support for distributed applications. IEEE Comput 33(3):68-76

19. Cugola G, Di Nitto E, Fuggetta A (2001) The JEDI event-based infrastructure and its application to the development of the OPSS WFMS. IEEE Trans SoftwEng 27(9):827-850

20. Cugola G, Di Nitto E (2001) Using a publish/subscribe middleware to support mobile computing. In: Advanced Topic Workshop on Middleware for Mobile Computing, (Heidelberg, Germany)

21. 3GPP website (2001) The Third Generation Partnership Project. http://www.3gpp.org/. Accessed 22 Feb 2018

22. Huh J-H (2017) PLC-based design of monitoring system for ICT-integrated vertical fish farm. HumCentComputInfSci 7(1):1-19

23. Park J-S, Jeong S-J, Lee Y-H, Kim K-S (2007) Implementation of rule based insurance product recommend and design system using fuzzy inference. J Soc e-Bus Stud 12(1):99-122 
24. Resnick P, Iacovou N, Suchak M, Bergstrom P, Riedl J (1994) GroupLens: an open architecture for collaborative filtering of netnews. In: Proceedings of ACM 1994 Conference on Computer Supported Cooperative Work, Chapel Hill, NC, pp 175-186

25. Lee O-J (2014) Hybrid Preference prediction technique using weighting based data reliability for collaborative filtering recommendation system. J Korea SocComputInf 19(5):61-69. 